\title{
Plasmons Reveal the Direction of Magnetization in Nickel Nanostructures
}

\author{
Ventsislav K. Valev, ${ }^{\dagger, *}$ Alejandro V. Silhanek, ${ }^{\ddagger}$ Werner Gillijns, ${ }^{\ddagger}$ Yogesh Jeyaram, ${ }^{\ddagger}$ Hanna Paddubrouskaya, ${ }^{\lessgtr}$ \\ Alexander Volodin, ${ }^{\S}$ Claudiu G. Biris, ${ }^{\perp}$ Nicolae C. Panoiu, ${ }^{\perp}$ Ben De Clercq, ${ }^{\|}$Marcel Ameloot, \\ Oleg A. Aktsipetrov, ${ }^{\text {} ा ~ V i c t o r ~ V . ~ M o s h c h a l k o v, ~}{ }^{\ddagger}$ and Thierry Verbiest ${ }^{\dagger}$ \\ ${ }^{\dagger}$ Molecular Electronics and Photonics, INPAC, `Superconductivity and Magnetism \& Pulsed Fields Group, INPAC, §Laboratory of Solid-State Physics and Magnetism, \\ Katholieke Universiteit Leuven, Celestijnenlaan 200 D, 3001, Leuven, Belgium, ${ }^{\perp}$ Department of Electronic and Electrical Engineering, University College London, \\ Torrington Place, London WC1E 7JE, United Kingdom, "University Hasselt and Transnational University Limburg, BIOMED, Diepenbeek, Belgium, and "Department of \\ Physics, Moscow State University, 11992 Moscow, Russia
}

$\mathrm{T}$ he development of nanophotonics, nanoelectronics, and nanomagnetics could result in new generations of devices, capable of storing and processing information at increasing speed and decreasing energy cost. However, the relationship between these three nanotechnologies is only beginning to be clarified. The influence of magnetic fields on surface plasmons combines aspects of photonics, electronics, and magnetics at the nanoscale. Surface plasmons are collective excitations of electrons under the influence of light's electromagnetic field, and in magnetic materials, these electrons can experience the effects of externally applied magnetic fields, as well. Recently, this area of research has attracted a lot of interest as it was shown that plasmons can be directed with externally applied magnetic fields. ${ }^{1}$ In order to further improve our understanding of the behavior of surface plasmons in magnetic media, we made use of a surface-specific magneto-optical technique, namely, magnetization-induced second harmonic generation (MSHG)..$^{2-4}$

Within the dipole approximation, optical second harmonic can originate only from regions of broken symmetry, for example, the surfaces and the interfaces of a centrosymmetric material. Due to symmetry considerations, MSHG has the same requirements for structural symmetry breaking. Consequently, MSHG can probe the magnetization at surfaces and interfaces down to the atomic monolayer, ${ }^{5}$ where plasmons are also present.

More specifically, a contrast in the MSHG intensity is recorded, depending on the sign of the magnetization in the material, and it has been reported that this contrast can be www.acsnano.org
ABSTRACT We have applied the surface-sensitive nonlinear optical technique of magnetization-induced second harmonic generation (MSHG) to plasmonic, magnetic nanostructures made of Ni. We show that surface plasmon contributions to the MSHG signal can reveal the direction of the magnetization. Both the plasmonic and the magnetic nonlinear optical responses can be tuned; our results indicate novel ways to combine nanophotonics, nanoelectronics, and nanomagnetics and suggest the possibility for large magneto-chiral effects in metamaterials.

KEYWORDS: metamaterial · plasmon · magnetism · nanostructures $\cdot$ second harmonic generation · nickel · nano-optics $\cdot$ nonlinear optics

reversed due to the presence of surface/ interface plasmons. ${ }^{6,7}$ The latter could also cause a general enhancement of the MSHG signal. Additionally, such enhancements were reported in ferromagnetic gratings ${ }^{8}$ and granular films. ${ }^{9,10}$ Yet, these are not the only ways in which magnetization and surface plasmons interact.

We show that surface plasmons can create asymmetries in the rotational dependence of the MSHG signal, which reveal the direction of the magnetization in G-shaped nanostructures made of nickel (see Figure 1). Our samples have a four-fold in-plane symmetry. Because the MSHG technique is symmetry-sensitive, rotating the sample yields a four-fold pattern. Surprisingly though, besides the main MSHG response peak, a "secondary peak" can be observed that renders the total MSHG pattern asymmetric. The position of the secondary peak, and hence the asymmetry, reverses with the direction of magnetization. This asymmetry originates in the interference of so-called "magnetic" nonlinear susceptibility tensor elements with surface plasmon contribution to the nonlinear susceptibility. The presence of magnetism and surface plasmon resonances, in our nanostructured samples, is demonstrated individually as
${ }^{*}$ Address correspondence to v.k.valev@fys.kuleuven.be.

Received for review July 20, 2010 and accepted December 02, 2010.

Published online December 9, 2010. $10.1021 / \mathrm{nn} 102852 \mathrm{~b}$

ㅇ 2011 American Chemical Society 


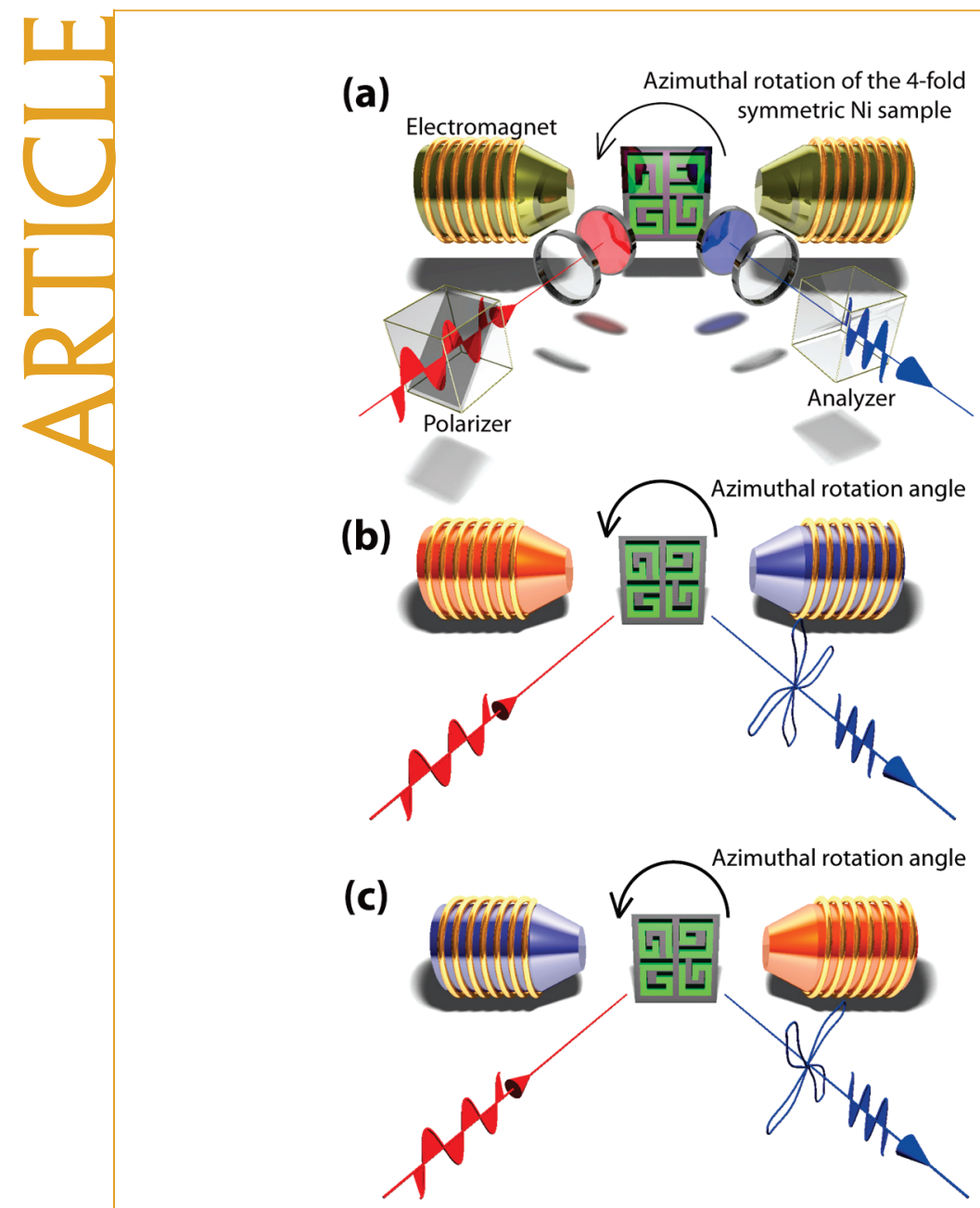

Figure 1. Magnetization-induced second harmonic generation is measured in four-fold symmetric magnetic, plasmonic nanostructures. (a) Schematic diagram of the experiment, whereby the sample is rotated in an externally applied magnetic field. (b) Upon rotating the sample in the presence of this magnetic field, the second harmonic generation intensity yields a four-fold pattern that is asymmetric; that is, it resembles a ratchet wheel. (c) Upon reversing the direction of the externally applied magnetic field, the direction of the ratchet wheel reverses, as well.

part of the sample preparation and characterization procedure.

Both Surface Plasmons and Magnetization Are Present in the Samples. The samples under investigation consist of a periodic array of G-shaped nanostructures, made of 25 $\mathrm{nm}$ thick Ni and covering a total area of $2.5 \times 2.5 \mathrm{~mm}^{2}$. The unit cell of the nanopattern is shown in the center of Figure 1a. These structures are $1 \mu \mathrm{m}$ long and 200 $\mathrm{nm}$ apart. Each line is $200 \mathrm{~nm}$ wide. Upon completing their preparation, the quality of these nanostructures is evaluated with respect to their magnetic and plasmonic responses.

The homogeneity of the magnetization in the $\mathrm{Ni}$ nanostructures is verified with magnetic force microscopy (MFM). In Figure 2a, the blue/yellow contrast indicates a typical MFM response for magnetization along the in-plane direction of an externally applied magnetic field $(B=25 \mathrm{mT})$. Similarly, in Figure $2 \mathrm{~b}$, the yellow/ blue contrast corresponds to a reversed magnetiza-
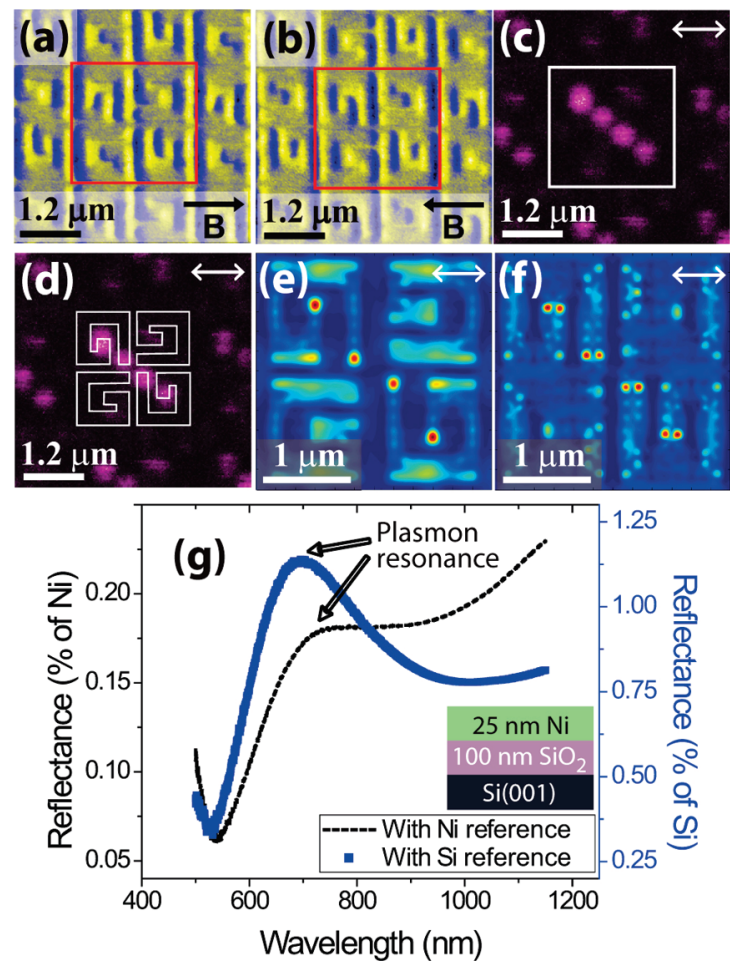

Figure 2. Magnetization-induced second harmonic generation is measured in four-fold symmetric magnetic, plasmonic nanostructures. $(a, b)$ Magnetic force microscope images of the sample structures. The yellow/blue contrast reveals typical in-plane magnetization for $B=+25$ and $-25 \mathrm{mT}$, respectively. (c) Second harmonic microscopy shows plasmonic local field enhancements at $800 \mathrm{~nm}$. The direction of polarization is indicated with an arrow. (d) Geometrical structures of the unit cell are superimposed on the SHG micrograph in order to illustrate the origin of the SHG hotspots. $(e, f)$ Spatial distribution of the intensity of the magnetic and electric fields, respectively, upon excitation of the sample with $800 \mathrm{~nm}$ light. (g) Plasmon resonance peaks revealed by spectroscopy. The sample thickness and substrate composition can be found in the inset.

tion. Both figures demonstrate high $\mathrm{Ni}$ deposition quality.

Regarding plasmons, it should be pointed out that such resonances have already been reported in nickel. ${ }^{11,12}$ In our samples, the presence of plasmons is directly evidenced by means of SHG microscopy images. Figure $2 c$ shows four hotspots within the unit cell of four Gs. While this unit cell is indicated with a red rectangle in Figure $1 \mathrm{~b}, \mathrm{c}$, it is indicated with a white rectangle in Figure $1 \mathrm{~d}$. For clarity, in Figure 2d, the geometry of the unit cell is reproduced over the SHG micrograph. In this manner, the origin of the hotspots is revealed. The hotspots themselves are due to localized field enhancements that result in localized SHG sources. The field enhancements are a consequence of localized plasmons in the Ni nanostructures, in agreement with numerical simulations of both the optical frequency magnetic (Figure 2e) and electric (Figure 2f) fields. Additionally, the reflectance spectra in Figure $2 \mathrm{~g}$ display broad plasmon resonances near $700 \mathrm{~nm}$. In order to rule out dispersion from the nickel or the sub- 
strate, the spectra are normalized both by a homogeneous Ni film and by the substrate.

MSHG Is a Surface-Specific Magneto-optical Technique. Having established the presence of both magnetization and plasmon resonances in the sample, it is now ready to be measured with MSHG. In Figure 1a, the experimental geometry with respect to the magnetic field is presented.

In linear optics, there is a relation of direct proportionality between the incident electric field $\mathbf{E}(\omega)$ at a frequency $\omega$ and the induced polarization in the material $\mathbf{P}(\omega)$. However, for intense electric fields, such as those produced by pulsed lasers, this linear relation does not hold anymore. The nonlinearity is attributed to the presence of higher harmonics of the induced polarization:

$\mathbf{P}(2 \omega), \mathbf{P}(3 \omega)$, etc. Each of these terms gives rise to light waves, which are emitted at the corresponding frequency. For second harmonic generation, in the electric dipole approximation, the induced polarization takes the following form:2,3,13

$$
\mathbf{P}(2 \omega)=\chi^{\mathrm{nm}}: \mathrm{E}(\omega) \mathrm{E}(\omega)
$$

where $\chi^{n m}$ is a third rank polar tensor that indicates the nonlinear susceptibility of a nonmagnetic (superscript "nm") material. In the presence of magnetization M, a new term appears:

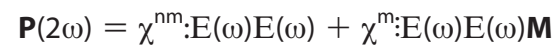

where $\chi^{\mathrm{m}}$ is a fourth rank axial tensor associated with the magnetization (superscript " $\mathrm{m}$ "). This new term gives rise to magnetization-induced SHG. Because the direction of $\mathbf{M}$ can be fixed by an external magnetic field, as in Figure 1a, $\chi^{\mathrm{m}}$ reduces to a third rank polar tensor and eq 2 can be written in a similar form to eq 1:

$$
\mathbf{P}(2 \omega)=\chi^{\text {eff: }}: \mathrm{E}(\omega) \mathrm{E}(\omega)
$$

where $\chi^{\text {eff }}$ is an effective nonlinear susceptibility that contains two types of tensor elements: "even" and "odd" in the magnetization. An even tensor element is one that does not change its sign upon reversing the direction of magnetization in the sample. At variance, an odd tensor element does change its sign upon reversing the direction of magnetization. The MSHG intensity then becomes

$$
I(2 \omega) \propto\left|\chi^{\text {even }} \pm \chi^{\text {odd }}\right|^{2} I^{2}(\omega)
$$

where it can be seen that, depending on the direction of magnetization, the MSHG signal can exhibit different intensity.

In our experiment, a magnetic field is applied in the plane of optical incidence (longitudinal configuration) and both the polarizer and the analyzer are set along the vertical direction $\left(S_{\mathrm{IN}}-S_{\mathrm{OUT}}\right)$. Under these conditions, for a sample with a four-fold symmetry (chiral or not), a single odd tensor element from the $\chi^{\text {eff }}$ tensor contribwww.acsnano.org

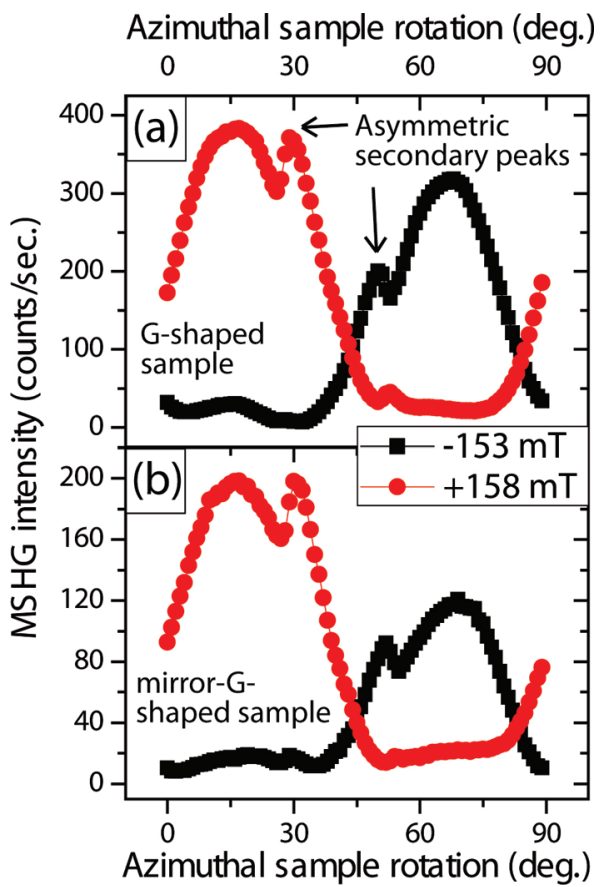

Figure 3. Upon rotating the four-fold sample, besides the expected four-fold response in the magnetization-induced second harmonic generation, secondary peaks appear which render the whole four-fold pattern asymmetric and gives it a direction of rotation, as in a ratchet wheel. The direction of rotation of this ratchet wheel is related to the direction of magnetization. This asymmetry is unaffected by chirality as illustrated by the graphs in (a) from the G-shaped samples and in (b) from the mirror-G-shaped samples.

utes to the signal. More specifically, this tensor element is $\chi_{y y y}^{\text {odd }}$, where the subscript indicates that the vertical (S) direction of polarization is along the $y$ axis of a Cartesian coordinate system on the sample. We can therefore write $\chi_{y y y}^{\text {odd }} \neq 0$. Consequently, according to eq 4, no magnetic contrast should be observed because $\chi^{\text {even }}=0$. However, as can be seen in Figure 3a, the magnetic contrast is dramatic, which demonstrates that, surprisingly, $\chi^{\text {even }} \neq 0$.

In our sample, the $\chi^{\text {even }}$ component is most likely due to a mixture of plasmon resonances and contributions from the "sides" of the nanostructures (surfaces that contain the direction of sample thickness). Neighboring sides of the nanostructures (those that face each other) generate second harmonic light with opposite phase (because of the opposite orientation of their local surface normals). Consequently, in small and densely packed nanoparticles, such contributions cancel. However, because in our nanostructures the sides are 200 $\mathrm{nm}$ apart, which is a sizable fraction of the $400 \mathrm{~nm}$ MSHG, and taking into account the angle of optical incidence, it can be seen that the sides can contribute to the signal. As for contributions from plasmon resonances, they are in fact the central topic of the following paragraphs.

The MSHG Response Is Asymmetric Due to Plasmons. Figure 3a shows the MSHG response as a function of the azimuthal sample rotation angle, for positive and negative 


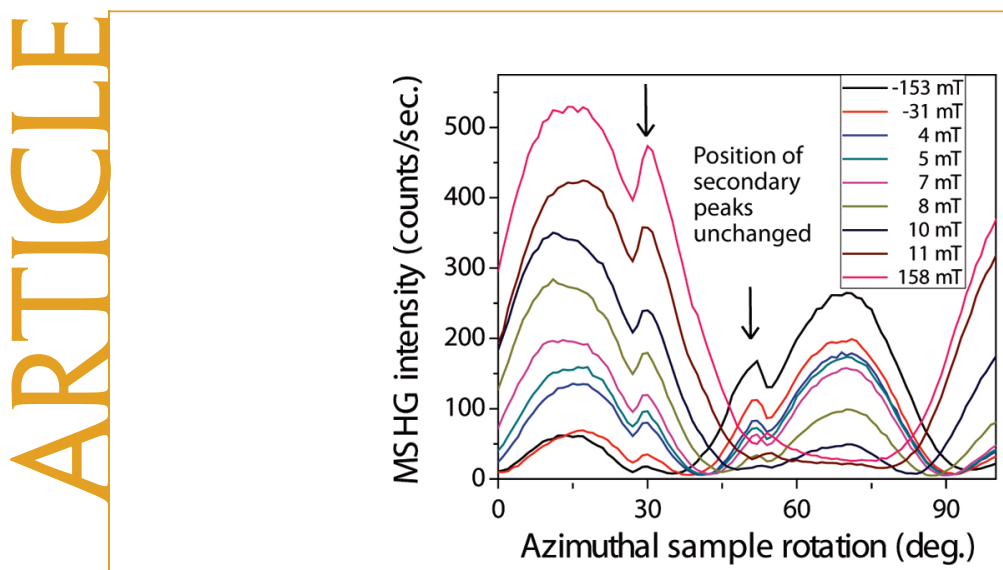

Figure 4. Secondary peaks themselves are not due to the value of the magnetic field as their position remains unaffected by it.

applied magnetic fields. The data cover the range from 0 to $90^{\circ}$, which is representative of the full sample rotation range, because of the four-fold symmetry of the samples. Upon applying a negative magnetic field, the MSHG shows a large main peak plus a secondary peak. Such a secondary peak is also present in the MSHG upon applying a positive magnetic field. These secondary peaks have a different position relative to the main peaks, and therefore, the resulting asymmetry indicates the sign of the applied magnetic field. What could be the origin of the secondary peaks?

Chirality, structural asymmetry, diffraction, magnetization, phase matching from the "vertical" sides of the nanostructures, and, indeed, plasmon excitations could all affect the data.

First, since asymmetric SHG has recently been reported from similarly shaped nanostructures made of gold ${ }^{14}$ chirality is a likely explanation for the presence of asymmetries in Figure 3a. However, in Figure 3b, it can be seen that upon imaging the sample with opposite handedness the results are identical to those in Figure 3a. This observation unambiguously rules out chirality as an explanation.

Next, the very large magnetic contrast in Figure 3a indicates that magnetic contributions to the SHG signal play an important role. Because of the complex geometry of the nanostructures, magnetic anisotropy can be affected at their edges and angles. Consequently, rotating the sample with respect to the direction of light's polarization could lead to local maxima of the magnetooptical effects. Furthermore, due to practical reasons, the two curves in Figure 3a were measured for slightly different absolute values of the magnetic field. Is this difference significant?

In Figure 4, the MSHG intensity is plotted as a function of sample azimuthal rotation angle for different values of the applied magnetic field. It is clear that, while the value of magnetic field changes, the position of the secondary peaks remains the same. This demonstrates that the difference in absolute value between a magnetic field of -153 and $+158 \mathrm{mT}$ is of no consequence

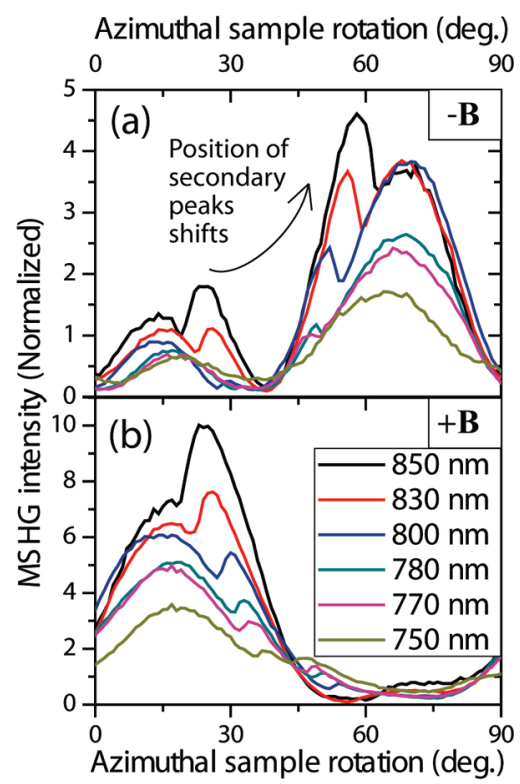

Figure 5. Position of the secondary peaks changes with wavelength. (a) MSHG intensity as a function of the azimuthal sample rotation upon applying a negative magnetic field (-153 mT). (b) MSHG intensity as a function of the azimuthal sample rotation upon applying a positive magnetic field (+158 mT). The MSHG signal was normalized by the MSHG spectral response of a homogeneous $\mathrm{Ni}$ thin film.

to the secondary peaks. In fact, because the peak at $30^{\circ}$ remains visible (even upon reversal of the magnetization), it cannot be attributed to magnetization or magnetic anisotropy. With magnetic behavior and chirality ruled out, we have to consider structural origin for the secondary peaks.

Since SHG is very sensitive to surface symmetry breaking effects and since the sample surface is clearly not isotropic, perhaps the secondary peaks could be attributed to the sample surface anisotropy. If that were the case, varying the laser wavelength should not affect the position of the secondary peaks.

In Figure 5, the MSHG response as a function of the sample azimuthal rotation angle is plotted for several wavelengths in the range of $750-850 \mathrm{~nm}$. The data were normalized by measuring the MSHG signals from a homogeneous nickel thin film at the same wavelengths. Figure 5a,b shows the MSHG response for negative and positive magnetic fields, respectively. In both panels, it is obvious that the position of the secondary peaks changes with wavelength. Because the structure remains the same, while the MSHG signal changes with wavelength, we can conclude that the secondary peaks do not originate in the surface anisotropy.

The dimensions of the nanostructures are close to the excitation wavelengths, and in our experiment, diffraction is observed. However, the data presented here were recorded in the zero-order of diffraction, which is undistinguishable from reflection. Henceforth, a wavelength dependence that is associated with diffraction cannot explain the data in Figure 5. 
Phase matching from contributions by the sides of the nanostructures (surfaces that contain the direction of sample thickness) is certainly affected by varying the wavelength. Nevertheless, taking into account the distance between these sides and the angle of optical incidence, it can easily be established that this particular type of signal contribution should diminish with increasing wavelength. This argument rules out contributions from the sides as an explanation.

Finally, plasmon resonances are present in these nanostructures (see Figure $2 \mathrm{~d}-\mathrm{g}$ ). These are wellknown for being wavelength-sensitive, but according to Figure $2 \mathrm{~g}$, a maximum in absorption occurs below $800 \mathrm{~nm}$. It should be noted though that these spectra were taken at normal incidence, while the MSHG was observed at an angle of incidence of $20^{\circ}$. Plasmon resonances are well-known for being dependent on the angle of optical incidence. Therefore, a shift in plasmon resonance for this incidence angle is the likeliest explanation for the origin of the secondary peaks.

In our samples, light waves couple to plasmon modes that depend on the geometry of the nanostructures. In Figure 3, rotating the sample changes the orientation of the structures with respect to the direction of optical polarization. Consequently, the total MSHG intensity can exhibit local maxima depending on whether or not plasmon modes are addressed along that particular direction.

The role of plasmons in the nonlinear optical response of similar G-shaped nanostructures made of gold has been reported before with respect to chirality. ${ }^{15}$ Nickel though is different in two important aspects: it is more absorbing than gold and it is magnetic. It should be noted that the plasmon enhancements visualized by the SHG microscopy image in Figure 2c are very similar to those in Figure 1 of ref 16, except that the figure in this article is less bright. This is not a trivial observation. An increase in laser power does not yield a brighter image. Figure 2c has been obtained with 0.98 $\mathrm{mW}$ incoming laser power, and at $1.23 \mathrm{~mW}$, the sample is irreversibly affected. This very low laser power threshold is attributed to the higher absorption of nickel. Because of that absorption, the plasmons have a limited propagation range which does not reflect the overall geometry of the nanostructures and especially their chirality (see Figure 2).

Magneto-Chiral Effect in Metamaterials? In conclusion, this study shows that plasmons can reveal the direction of magnetization in G-shaped nanostructures made of nickel. Previous studies showed that plasmons can reveal the handedness in G-shaped nanostructures made of gold. It is therefore very likely that G-shaped nanostructures made of gold and nickel layers exhibit magneto-chiral effects. It has been predicted ${ }^{17}$ and experimentally verified ${ }^{18,19}$ that chirality can lead to negative refractive index without the need for negative permittivity or permeability. We propose that a large magneto-chiral effect might allow the possibility to switch the sign of the refractive index with an externally applied magnetic field.

\section{METHODS}

Sample Preparation. The samples were prepared on a $\mathrm{SiO}_{2}(100$ $\mathrm{nm}) / \mathrm{Si}(001)$ substrate by electron beam lithography with a double resist layer PMMA/Co-MMA (polymethyl methacrylate) in order to facilitate the subsequent lift-off. The Ni deposition was carried out in a molecular beam epitaxial system at a pressure of $\sim 5 \times 10^{-9} \mathrm{mbar}$ and a rate of $\sim 0.2 \AA / \mathrm{s}$. Lift-off was then performed with boiling acetone.

Experimental Characterization. For the MFM measurements, we used a commercial scanning probe system (Park Scientific Instruments, M5). The SHG microscopy images were obtained with a confocal laser scanning microscope ${ }^{20}$ (Zeiss LSM 510) using a femtosecond pulsed Ti:sapphire laser (DeepSee Mai-Tai). The laser pulses at $800 \mathrm{~nm}$ were focused on the sample to a spot that measures $330 \mathrm{~nm}$ in the direction of the polarization and $440 \mathrm{~nm}$ in the perpendicular direction. Reflectance spectra were taken with a Vertex 80v FT-IR spectrometer, coupled to a Hyperion microscope. MSHG experiments were performed at $800 \mathrm{~nm}$, with a Mai-Tai femtosecond laser system. The fundamental light, having a power of $85 \mathrm{~mW}$, was focused on the sample to a spot approximately $40 \mu \mathrm{m}$ in diameter. The angle of optical incidence on the sample was $20^{\circ}$. The generated $400 \mathrm{~nm}$ photons were then filtered through a BG39 filter that blocks the fundamental beam and were detected with a cooled photomultiplier tube. Finally, the intensity of the $400 \mathrm{~nm}$ signal was evaluated with a photon counter. For the purpose of our experiments, the sample was mounted on a motorized rotation stage, which enables the measurement of MSHG (photons/s) at different rotation angles.

Numerical Simulations. Numerical simulations were performed with commercial software, based on the rigorous coupled-wave analysis method, numerical convergence being reached if 18 dif- fraction orders are used for each transverse dimension. We assumed that the dielectric constant of $\mathrm{Ni}$ is described by the Lorentz-Drude model, with the interband effects being characterized by a superposition of four Lorentzians.

Acknowledgment. We acknowledge financial support from the Fund for scientific research Flanders (FWO-V), the University of Leuven (GOA), Methusalem Funding by the Flemish government, and the Belgian Inter-University Attraction Poles IAP Programmes. V.K.V., A.V.S., and W.G. are grateful for the support from the FWO-Vlaanderen. C.G.B. and N.C.P. acknowledge support from the EPSRC. O.A.A. is partly supported by the Russian Foundation for Basic Research. B.D.C. is grateful to the IWT.

\section{REFERENCES AND NOTES}

1. Temnov, V. V.; Armelles, G.; Woggon, U.; Guzatov, D; Cebollada, A.; Garcia-Martin, A.; Garcia-Martin, J.-M.; Thomay, T.; Leitenstorfer, A.; Bratschitsch, R. Active Magneto-Plasmonics in Hybrid Metal-Ferromagnet Structures. Nat. Photonics 2010, 4, 107-111.

2. Kirilyuk, A.; Rasing, Th. Magnetization-Induced-SecondHarmonic Generation from Surfaces and Interfaces. J. Opt. Soc. Am. B 2005, 22, 148-167.

3. Fiebig, M.; Pavlov, V. V.; Pisarev, R. V. Second-Harmonic Generation as a Tool for Studying Electronic and Magnetic Structures of Crystals: Review. J. Opt. Soc. Am. B 2005, 22, 96-118.

4. McGilp, J. F.; Carroll, L.; Fleischer, K.; Cunniffe, J. P.; Ryan, S. Magnetic Second-Harmonic Generation from Interfaces and Nanostructures. J. Magn. Magn. Mater. 2010, 322, 1488-1493. 
5. Valev, V. K.; Kirilyuk, A.; Rasing, Th.; Dela Longa, F.; Kohlhepp, J. T.; Koopmans, B. Oscillations of the Net Magnetic Moment and Magnetization Reversal Properties of the Mn/Co Interface. Phys. Rev. B 2007, 75, 012401.

6. Tessier, G.; Malouin, C.; Georges, P.; Brun, A.; Renard, D.; Pavlov, V. V.; Meyer, P.; Ferré, J.; Beauvillain, P.

Magnetization-Induced Second-Harmonic Generation Enhanced by Surface Plasmons in Ultrathin $\mathrm{Au} / \mathrm{Co} / \mathrm{Au}$ Metallic Films. Appl. Phys. B: Laser Opt. 1999, 68, 545-548.

7. Pavlov, V. V.; Tessier, G.; Malouin, C.; Georges, P.; Brun, A.; Renard, D.; Meyer, P.; Ferré, J.; Beauvillain, P. Observation of Magneto-optical Second-Harmonic Generation with Surface Plasmon Excitation in Ultrathin $\mathrm{Au} / \mathrm{Co} / \mathrm{Au}$ Films. Appl. Phys. Lett. 1999, 75, 190-192.

8. Newman, D. M.; Wears, M. L.; Matelon, R. J.; Hooper, I. R. Magneto-optic Behaviour in the Presence of Surface Plasmons. J. Phys.: Condens. Matter 2008, 20, 345230.

9. Aktsipetrov, O. A.; Murzina, T. V.; Kim, E. M.; Kapra, R. V.; Fedyanin, A. A.; Inoue, M.; Kravets, A. F.; Kuznetzova, S. V.; Ivanchenko, M. V.; Lifshits, V. G. Magnetization-Induced Second- and Third-Harmonic Generation in Magnetic Thin Films and Nanoparticles. J. Opt. Soc. Am. B 2005, 22, 138147.

10. Murzina, T. V.; Misuryaev, T. V.; Kravets, A. F.; Gudde, J.; Schuhmacher, D.; Marowsky, G.; Nikulin, A. A.; Aktsipetrov, O. A. Nonlinear Magneto-optical Kerr Effect and Plasmon-Assisted SHG in Magnetic Nanomaterials Exhibiting Giant Magnetoresistance. Surf. Sci. 2001, 482485, 1101-1106.

11. Melle, S.; Menéndez, J. L.; Armelles, G.; Navas, D.; Vázquez, M.; Nielsch, K.; Wehrspohn, R. B.; Gösele, U. Magnetooptical Properties of Nickel Nanowire Arrays. Appl. Phys. Lett. 2003, 83, 4547-4549.

12. González-Díaz, J.; García-Martín, A.; Armelles, G.; Navas, D.; Vázquez, M.; Nielsch, K.; Wehrspohn, R.; Gösele, U. Enhanced Magneto-optics and Size Effects in Ferromagnetic Nanowire Arrays. Adv. Mater. 2007, 19, 2643-2647.

13. Rasing, Th. Nonlinear Magneto-optics. J. Magn. Magn. Mater. 1997, 175, 35-50.

14. Valev, V. K.; Silhanek, A. V.; Verellen, N.; Gillijns, W.; Van Dorpe, P.; Vandenbosch, G. A. E.; Aktsipetrov, O. A. Moshchalkov, V. V.; Verbiest, T. Asymmetric Second Harmonic Generation from Chiral G-Shaped Gold Nanostructures. Phys. Rev. Lett. 2010, 104, 127401.

15. Valev, V. K.; Smisdom, N.; Silhanek, A. V.; De Clercq, B.; Gillijns, W.; Ameloot, M.; Moshchalkov, V. V.; Verbiest, T. Plasmonic Ratchet Wheels: Switching Circular Dichroism by Arranging Chiral Nanostructures. Nano Lett. 2009, 9, 3945-3948.

16. Valev, V. K.; Silhanek, A. V.; Smisdom, N.; De Clercq, B.; Gillijns, W.; Aktsipetrov, O. A.; Ameloot, M.; Moshchalkov, V. V.; Verbiest, T. Linearly Polarized Second Harmonic Generation Microscopy Reveals Chirality. Opt. Express 2010, 18, 8286-8293.

17. Pendry, J. B. A Chiral Route to Negative Refraction. Science 2004, 306, 1353-1355.

18. Zhang, S.; Park, Y.-S.; Li, J.; Lu, X.; Zhang, W.; Zhang, X. Negative Refractive Index in Chiral Metamaterials. Phys. Rev. Lett. 2009, 102, 023901.

19. Plum, E.; Zhou, J.; Dong, J.; Fedotov, V. A.; Koschny, T.; Soukoulis, C. M.; Zheludev, N. I. Metamaterial with Negative Index Due to Chirality. Phys. Rev. B 2009, 79, 035407.

20. Gielen, E.; Smisdom, N.; van de Ven, M.; De Clercq, B.; Gratton, E.; Digman, M.; Rigo, J.-M.; Hofkens, J.; Engelborghs, Y.; Ameloot, M. Measuring Diffusion of Lipidlike Probes in Artificial and Natural Membranes by Raster Image Correlation Spectroscopy (RICS): Use of a Commercial Laser-Scanning Microscope with Analog Detection. Langmuir 2009, 25, 5209-5218. 